\title{
Performance of hemodynamic stability parameters as predictors of ectopic pregnancy, with emphasis on shock index
}

\author{
Bijay Khan*, Mayoukh Kumar Chakraborty, Ranjan Basu
}

Department of Obstetrics and Gynecology, KPC Medical College, Kolkata, West Bengal, India

Received: 01 February 2022

Revised: 16 February 2022

Accepted: 17 February 2022

\author{
*Correspondence: \\ Dr. Bijay Khan, \\ E-mail: drbkhan2015@gmail.com
}

Copyright: ( $\subseteq$ the author(s), publisher and licensee Medip Academy. This is an open-access article distributed under the terms of the Creative Commons Attribution Non-Commercial License, which permits unrestricted non-commercial use, distribution, and reproduction in any medium, provided the original work is properly cited.

\begin{abstract}
Background: A study was conducted to determine the utility of shock index in the diagnosis of acute rupture ectopic pregnancy (EP).

Methods: A retrospective review of all ruptured ectopic pregnancy at KPC medical college and hospital a tertiary private health care centre was performed for two years (May 2018 to April 2020). During this study, we used data of 35 cases of EP among 1150 pregnant ladies admitted from time to time under emergency.

Results: The mean age of the study population was $29.49 \pm 0.74$ (mean \pm SEM) years (range 22-38 years) and mean gestational period of pregnancy was 42.2 \pm 2.0 days (range 28-90 days). The commonest presentation of clinical symptoms associated with EC was abdominal pain (91\%), vaginal bleeding (89\%), amenorrhea (86\%), dizziness/fainting attack $(17 \%)$ and shock $(68 \%)$. In majority $(92.3 \%)$ of the patients the shock index was $>0.70$ with an average of $0.9 \pm 0.03$. In $60.7 \%$ of the cases the amount of haemoperitoneum found at laparatomy was $>1000 \mathrm{ml}$. It was observed that most patients with EP had hemoperitoneum above $1000 \mathrm{ml}(1140 \pm 113.6)$.

Conclusions: Pulse rate or systolic blood pressure alone are not a good predictor to access the amount of blood loss in EP. But when they were used to calculate the SI (shock index), it was more accurate and simpler predictor for diagnosis of EP. SI may be used in guiding clinicians in providing appropriate management for EP patients especially in lowresource settings or hospitals with limited facilities.
\end{abstract}

Keywords: Ectopic pregnancy, Emergency, Haemorrhage, Shock index

\section{INTRODUCTION}

EP are associated with high morbidity and mortality across the globe. The incident of EP is now increasing both apparent and actual because of pregnancy at increase age of mother, artificial reproductive technique, pelvic inflammatory diseases and proper diagnosis of cases. An early detection for EP is a most prognostic factor of the better outcome. EP occurs when a fertilized ovum implants on any sites other than the normal site of the endometrial of the uterus. EPs can be life threatening gynecological emergency and accounts for about $10-15 \%$ of all pregnancy associated deaths and leading cause of firsttrimester maternal death. ${ }^{1-2}$ It was studied by Murry et al that about $6-16 \%$ of pregnant women, who were rushed to emergency department in the first trimester for bleeding with pain or both, have an EP. ${ }^{3}$ The pathophysiology of haemorrhage in ectopic pregnancy is known and its ultimate consequence of grade III or IV haemorrhagic shock due to more than $30 \%$ loss of blood volume and uncompensated mechanism. If it is detected in early stage it may be reversible. Although advances in diagnosis have led to decreased morbidity and mortality, yet there is a further need to device means of early detection and 
treatment. Predicting tools and risk stratification algorithms may play a pivotal role in evaluation of serious illness like EP. ${ }^{3}$ Though there are several modern modes of investigations available for diagnosis of EP yet clinical parameter judgement is essential for early diagnosis and management of EC. The modern gadgets are still not available in all health set-ups in less developed parts of the world including India. Patients with ruptured ectopic pregnancy could present with several sign and symptoms including haemodynamic parameters. In the present paper we examined performances of various hemodynamic stability parameters as predictors of ectopic pregnancy, with emphasis on shock index. We reported sensitivities, specificities and positive and negative predictive values for the primary and secondary outcomes.

\section{METHODS}

\section{Study design and parameters}

During this retrospective study, we used data of 35 cases of EP among 1150 pregnant ladies admitted from time to time under emergency during May 2018 April 2020 at KPC medical college, Kolkata, India. For this study only those cases were included in which a diagnosis of ruptured EP was made clinically and some amount of haemoperitoneum was obtained during the emergency laparotomy. The cases in which haemoperitoneum was not found at laparotomy were excluded. All the 35 cases identified had complete information for analysis. The age structure and other demographic information were included in Figure 1 and Table 1. Data were collected and transferred to a proforma already outlined in the beginning for the study. The data extracted included patients' demographic characteristics, preoperative vital signs, the amount of haemoperitoneum obtained during surgery, blood transfusion and complications. For accurate determination of the vital signs were recorded during the immediate pre-operative period. During laparotomy, any encountered haemoperitoneum was suctioned out and its quantity was measured both volumetrically and through visual estimation of socked gauge and linens. For each set of pulse rate (heart rate) and systolic blood pressure, shock index was calculated by dividing the pulse rate with the systolic blood pressure. Numbers and simple percentages were used to report proportions of the vital signs and shock index. The correlation between the study variables and the selected outcomes including the amount of haemoperitoneum, blood transfusion requirement and various complications were obtained using the raw scale data and then stepwise logistic regression analysis was used to construct a predictive model for these outcomes using predefined values of the variables. As hypotension was a blood pressure of 90/60 $\mathrm{mm}$ hg and below and tachycardia, pulse rate of 100 and above, these predefined values were taken as pulse rate of $>100$, systolic blood pressure of $<90 \mathrm{mmHg}$ and, diastolic blood pressure of $<60 \mathrm{mmHg}$. The predefined value of shock index was taken as $>0.70$.
We explored the usefulness of several hemodynamic stability parameters including SI as predictors of EP. The covariates considered in our analysis included haemoglobin level, blood transfusion requirement, systolic blood pressure $(\mathrm{mm} \mathrm{Hg})$, diastolic blood pressure ( $\mathrm{mm}$ $\mathrm{Hg}$ ), pulse rate and SI. Frequencies of clinical symptoms were selected as per common observation modified from Lawani et al SI calculated as an average heart rate/systolic blood pressure, had previously been shown to identify high risk patients in different ailments. ${ }^{5-7}$ Additional information abstracted included, age of the patient and mean gestational period, details of obstetrics, gynaecological, medical and surgical history. Also bedside urinary $\beta$-HCG test was conducted to evaluate the patients for pregnancy. All EP documented in the study were validated by pathological examination.

\section{Statistical analysis}

For easy interpretation, vital parameters (SBP, DBP, haemoglobin level, blood transfusion data, SI) were categorized into binary categorical variables (Table 2). The results are expressed as mean \pm SEM throughout the study. Further various hemodynamic stability parameters including haemoglobin level, blood transfusion requirement, SBP, DBP, pulse rate, SI were correlated with respect to each other and predictive values were calculated using Spearman's rank correlation coefficient. SPSS 26.0 for Windows was used for statistical analysis (SPSS Inc., Chicago, IL, USA). Statistical significance was accepted when the $\mathrm{p}$ value was lower than 0.05 .

\section{RESULTS}

The study COHORT consisted of 35 patients admitted to hospital due to EP, with a mean \pm SEM age of $29.49 \pm 0.74$ years (range 22-38 years) and mean gestational period of pregnancy was $42.2 \pm 2.0$ days (range 28-90 days) (Figure 1).

The threshold values of all recorded vital signs/hemodynamic stability parameters were listed in Table 2. The frequency of clinical symptoms associated with EC is shown in Table 3. The commonest presentation was abdominal pain (91\%), 89\% had vaginal bleeding, $86 \%$ presented with amenorrhea, $17 \%$ presented with dizziness/fainting attack and $68 \%$ presented in shock. Other less common symptoms included shoulder-tip-pain (32\%) and diarrhoea (29\%). Hemodynamic stability parameters including the heart rate, SBP and SI (heart rate/SBP) have been included in Table 4.

The results of the Spearman's rho correlation showed that there was a significant negative linear relationship $(p<0.05)$ between the various hemodynamic stability parameters (Table 5). The correlation of shock index with the amount of blood loss and Hb levels highly significant $(\mathrm{p}<0.01)$ and it was also significantly associated with gestational age and blood transfusion. 
Table 1: Demographic characteristics of patients received for study of EP.

\begin{tabular}{|c|c|c|c|}
\hline S. No. & Demographic characteristics & Frequency & Percentage (\%) \\
\hline \multirow{5}{*}{1.} & Educational status & & \\
\hline & Illiterate & 2 & 5 \\
\hline & Less than HS & 12 & 37 \\
\hline & Graduate & 18 & 47 \\
\hline & Higher & 3 & 8 \\
\hline \multirow{4}{*}{2} & Socio-economic status & & \\
\hline & Low income & 23 & 63 \\
\hline & Medium income & 9 & 27 \\
\hline & High income & 3 & 8 \\
\hline \multirow{3}{*}{3.} & Habitat & & \\
\hline & Rural & 24 & 63 \\
\hline & Urban & 11 & 29 \\
\hline
\end{tabular}

Table 2: Threshold values of haemodynamic parameters.

\begin{tabular}{|ll|}
\hline Parameters evaluated & Threshold values \\
\hline Haemoglobin level (g/dl) & 10 \\
\hline Blood transfusion requirement & $\geq 2$ \\
\hline SBP $(\mathbf{m m H g})$ & $\geq 100$ \\
\hline DBP $(\mathbf{m m H g})$ & $\leq 60$ \\
\hline Pulse rate (per min) & 100 \\
\hline SI & 0.7 \\
\hline
\end{tabular}

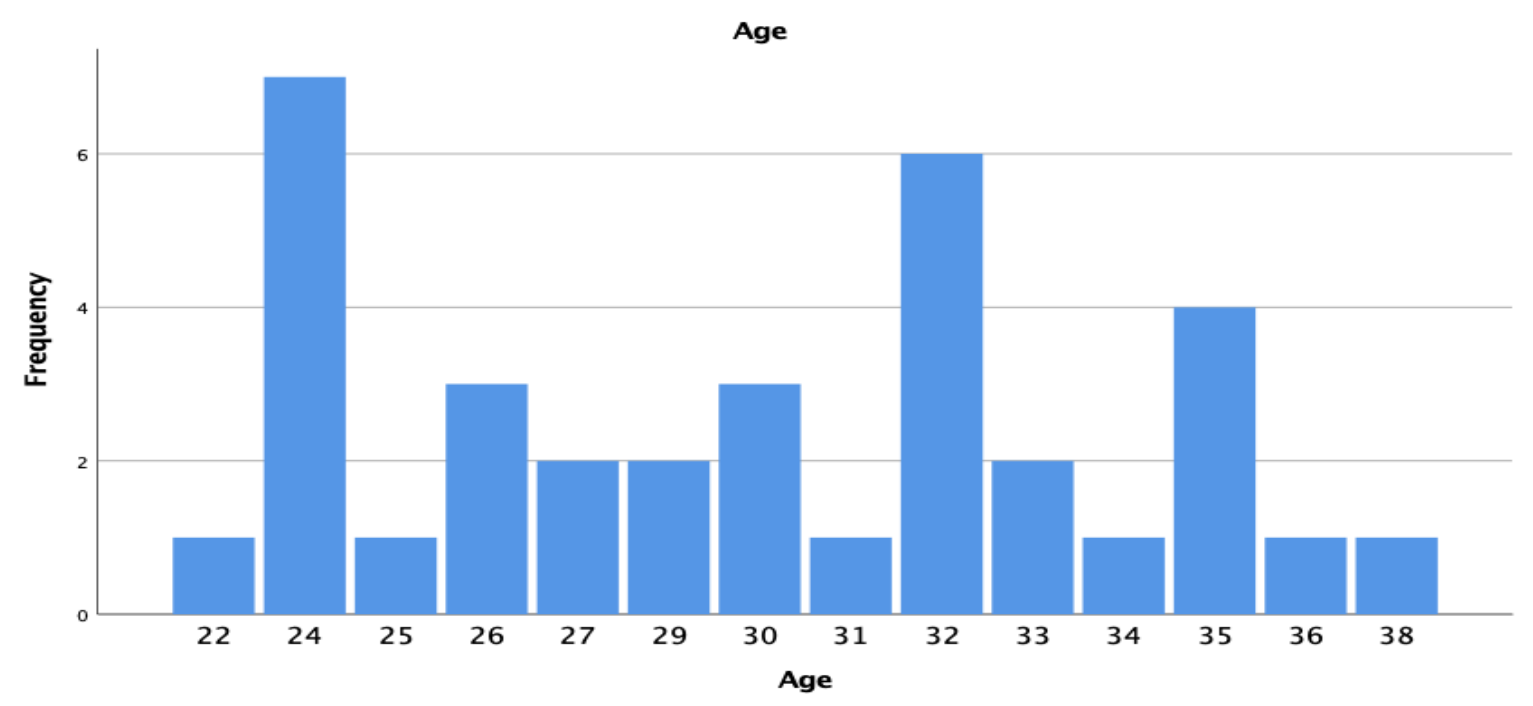

Figure 1: Age structure of patients received for study of EP.

Table 3: Frequency of clinical symptoms of patients with EP.

\begin{tabular}{|l|c|}
\hline Symptoms & Frequency \\
\hline Abdominal pain & 91 \\
\hline Amenorrhea & 86 \\
\hline Vaginal bleeding & 89 \\
\hline Fainting attacks/dizziness & 17 \\
\hline Shock & 68 \\
\hline Shoulder-tip-pain & 32 \\
\hline Diarrhoea & 29 \\
\hline
\end{tabular}


Table 4: Performance of hemodynamic stability parameters as predictors of EP.

\begin{tabular}{|lll|l|}
\hline Parameters evaluated $(\mathbf{n}=\mathbf{3 5})$ & & Frequency & Percentage \\
\hline \multirow{2}{*}{ Haemoglobin level } & Low $(<10)$ & 30 & 85.7 \\
\cline { 2 - 4 } \multirow{2}{*}{ Blood transfusion requirement } & Normal $(\geq 10)$ & 5 & 14.3 \\
\hline \multirow{2}{*}{ SBP } & Yes & 29 & 82.9 \\
\cline { 2 - 4 } & No & 6 & 17.1 \\
\hline \multirow{2}{*}{ DBP } & Normal $(\geq 100)$ & 22 & 62.9 \\
\cline { 2 - 4 } & Low $(<100)$ & 13 & 35.1 \\
\hline \multirow{3}{*}{ Pulse rate } & Normal $(\geq 60)$ & 27 & 77.1 \\
\cline { 2 - 4 } & Low $(<60)$ & 8 & 22.9 \\
\hline \multirow{2}{*}{ Shock Index } & High $(\geq 100)$ & 14 & 40 \\
\cline { 2 - 4 } & Normal $(99-61)$ & 21 & 60 \\
\cline { 2 - 4 } & Low $(\leq 60)$ & 0 & 0 \\
\cline { 2 - 4 } & High $(\geq 0.7)$ & 33 & 94.3 \\
\cline { 2 - 4 } & Low $(<0.7)$ & 2 & 5.7 \\
\hline
\end{tabular}

Table 5: Correlation matrix obtained from Spearman's rho correlation study.

\begin{tabular}{|c|c|c|c|c|c|c|c|}
\hline \multicolumn{3}{|l|}{ Correlation } & $\begin{array}{l}\text { Shock } \\
\text { Index }\end{array}$ & $\begin{array}{l}\text { Blood } \\
\text { Loss }\end{array}$ & $\begin{array}{l}\text { Gestational } \\
\text { age }\end{array}$ & $\begin{array}{l}\text { Blood } \\
\text { Transfusion }\end{array}$ & $\begin{array}{l}\text { Hb } \\
\text { level }\end{array}$ \\
\hline \multirow{15}{*}{$\begin{array}{l}\text { Spearman's } \\
\text { rho }\end{array}$} & \multirow{3}{*}{ SI } & Correlation coefficient & 1.000 & $.454 * *$ & $.360 *$ & $.391 *$ & $-.557 * *$ \\
\hline & & Sig. (2-tailed) & .0 & .006 & .034 & .020 & .001 \\
\hline & & $\mathrm{N}$ & 35 & 35 & 35 & 35 & 35 \\
\hline & \multirow{3}{*}{ Blood loss } & Correlation coefficient & $.454 * *$ & 1.000 & .081 & $.343^{*}$ & $-.450 * *$ \\
\hline & & Sig. (2-tailed) & .006 & & .643 & .044 & .007 \\
\hline & & $\mathrm{N}$ & 35 & 35 & 35 & 35 & 35 \\
\hline & \multirow{3}{*}{$\begin{array}{l}\text { Gestational } \\
\text { age }\end{array}$} & Correlation coefficient & $.360^{*}$ & .081 & 1.000 & $.538 * *$ & -.318 \\
\hline & & Sig. (2-tailed) & .034 & .643 & .0 & .001 & .063 \\
\hline & & $\mathrm{N}$ & 35 & 35 & 35 & 35 & 35 \\
\hline & \multirow{3}{*}{$\begin{array}{l}\text { Blood } \\
\text { transfusion }\end{array}$} & Correlation coefficient & $.391 *$ & $.343^{*}$ & $.538 * *$ & 1.000 & $-.646 * *$ \\
\hline & & Sig. (2-tailed) & .020 & .044 & .001 & .0 & .000 \\
\hline & & $\mathrm{N}$ & 35 & 35 & 35 & 35 & 35 \\
\hline & \multirow{3}{*}{ Hb level } & Correlation coefficient & $-.557 * *$ & $-.450 * *$ & -.318 & $-.646 * *$ & 1.000 \\
\hline & & Sig. (2-tailed) & .001 & .007 & .063 & .000 & \\
\hline & & $\mathrm{N}$ & 35 & 35 & 35 & 35 & 35 \\
\hline
\end{tabular}

**Correlation is significant at the 0.01 level (2-tailed); *correlation is significant at the 0.05 level (2-tailed).

\section{DISCUSSION}

This study showed that all the vital signs/hemodynamic stability parameters had a strong correlation among themselves. The SI was observed to be a useful parameter in the management of patients with ruptured EP. Similar to our studies, Hick et al reported significant correlation between vital signs and EP. ${ }^{8}$

The mean age of 29.49 years in the study population was similar to other studies. ${ }^{8,9}$ It was observed that most patients with EP had hemoperitoneum (intra-abdominal blood loss) above $1000 \mathrm{ml}(1140 \pm 113.6)$.

In our study $94.3 \%$ cases of EP had $\mathrm{SI} \geq 0.7$ with an average of $0.9 \pm 0.03$ which was similar to the studies by Geidam et al. ${ }^{9}$ SI was first introduced in 1967 as a simple and effective way to measure the degree of hypovolemia in the state of shock for primary diagnosis of disease severity, identify high-risk patients and predict morbidity. ${ }^{7}$ Berger et al while predicting SI relation with stroke patients, reported that compared with patients with SI of 0.5 to 0.7 , patients with SI $>0.7$ had worse outcomes. ${ }^{6}$ Majority (60\%) of the patients had pulse rate was between 61-99 (normal) while only $40 \%$ patients had pulse rate $\geq 100$. So pulse rate alone was not a good predictor to access the amount of blood loss in EP. But when it was used to calculate the SI in combination with SBP it was more accurate and simple predictor for diagnosis of EP.

In patients with EP morbidity and mortality results from the associated haemorrhage. So to reduce this morbidity and mortality in pregnant women, clinicians should identify those patients likely to develop complications due to haemorrhage in EP to initiate early treatment. Though we accessed the amount of blood loss by the vital signs particularly the heart rate and blood pressure separately, SI 
was a more sensitive tool. It evaluated the haemorrhage more accurately because the left ventricular work was dependent on cardiac output and volume status causing SI to be directly affected by hyopvolaemia. ${ }^{7}$

SI had been known to serve as an easily available bedside assessment tool to identify high risk patients with variety of causes including trauma, haemorrhage, myocardial infarction, pulmonary embolism, sepsis and ruptured EP. ${ }^{3}$ Thus guiding clinicians in providing appropriate management for these patients especially in low-resource settings or hospitals with limited facilities.

\section{Limitations}

The major limitation of the study was that the data presented in the paper concentrate on one selected hospital in Kolkata, India. Also the study was conducted on limited number of cases from one region. More similar studies under different climatic, racial, geographical regions taking into account possible variability may be useful. Hence the work may be replicated by other researchers for more validation.

\section{CONCLUSION}

Our study shows that pulse rate or SBP singly are not a good predictor to access the amount of intra-abdominal blood loss in EP. However, when they were used to calculate the SI, it was more accurate and simpler predictor for diagnosis of EP and leads to better assessment of the patients general condition and early resuscitative measures can be taken accordingly. SI may be used in guiding clinicians, particularly in less-developed parts of the world with less medical facilities, to provide appropriate and quicker management for EP patients.

\section{Funding: No funding sources}

Conflict of interest: None declared

Ethical approval: The study was approved by the Institutional Ethics Committee

\section{REFERENCES}

1. Stremick JK, Couperus K, Ashworth SW. Ruptured tubal ectopic pregnancy at fifteen weeks gestational age. Clin Pract Cases Emerg Med. 2019;3(1):62-4.

2. Sara HG, Uzelac PS. Early pregnancy risks. In: DeCherney AH, Nathan L, Goodwin MT, Laufer N, eds. Current diagnosis and treatment: obstetrics and gynecology. 10th ed. Columbus (OH): McGraw-Hill; 2007: 259-72.

3. Murray H, Baakdah H, Bardell $\mathrm{T}$, Tulandi $\mathrm{T}$. Diagnosis and treatment of ectopic pregnancy. Canadian Med Assoc J. 2005;173(8):905-12.

4. Koch E, Lovett S, Nohiem T, Riggs RA, Rech MA. Shock index in the emergency department: utility and limitations. Open Access Emerg Med. 2019;11:17999.

5. Lawani OL, Anozie OB, Ezeonu PO. Ectopic pregnancy: a life-threatening gynecological emergency. Int J Women Health. 2013;5:515-21.

6. Berger, Green J, Horeczko T, Hagar Y, Garg N, Suarez A, et al. Shock index and early recognition of sepsis in the emergency department: pilot study tony west. J Emerg Med. 2019;14(2):168-74.

7. Maheshwari K, Nathanson BH, Munson SH, Hwang S, Yapici HO, Stevans M, et al. Abnormal shock index exposure and clinical outcomes among critically ill patients: a retrospective COHORT analysis. J Crit Care. 2020;57:5-12.

8. Hick JL, Rodgerson JD, Heegaard WG, Sterner S. Vital signs fail to correlate with hemoperitoneum from ruptured ectopic pregnancy. Am J Emerg Med. 2001;19(6):488-91.

9. Geidam AD, Audu BM, Mairiga AG. How useful is shock index in the management of ruptured ectopic pregnancy. BOMJ. 2007;4(1):5-9.

Cite this article as: Khan B, Chakraborty MK, Basu R. Performance of hemodynamic stability parameters as predictors of ectopic pregnancy, with emphasis on shock index. Int J Reprod Contracept Obstet Gynecol 2022;11:887-91. 